\title{
Histopathologic Distribution of Appendicitis at Dr. Hasan Sadikin General Hospital, Bandung, Indonesia, in 2012
}

\author{
Tara Zhafira, ${ }^{1}$ Herry Yulianti, ${ }^{2}$ Maman Wastaman ${ }^{3}$ \\ ${ }^{1}$ Faculty of Medicine Universitas Padjadjaran, ${ }^{2}$ Department of Anatomical Pathology Faculty of \\ Medicine, Universitas Padjadjaran/Dr. Hasan Sadikin General Hospital, Bandung, ${ }^{3}$ Department \\ of Surgery Faculty of Medicine Universitas Padjadjaran/Dr. Hasan Sadikin General Hospital, \\ Bandung
}

\section{Abstract}

Background: Appendicitis is a medical emergency and a common cause of emergency surgeries worldwide Its frequency is varied based on many factors, including age and sex. Histopathologic examination is a gold standard for diagnosis, and complications like gangrene formation and perforation lead to high mortality and morbidity in almost all age groups. This study was conducted to describe the distribution pattern of appendicitis according to age, sex, and histopathologic type.

Methods: This cross-sectional study was carried out in the Department of Pathology Anatomy, Dr. Hasan Sadikin General Hospital, Bandung, Indonesia, from August-October 2013. Secondary data were obtained from medical records of January 1st to December 31st, 2012. A total of 503 out of 516 cases were included to be reviewed. Age, sex, and histopathologic type from medical records were then evaluated. Any specific case and perforation were also noted.

Results: Data showed the highest prevalence of appendicitis occurred in the 10- 19 age group (28.4\%) and in the female group (52.3\%). Acute appendicitis was more common than chronic appendicitis in both sexes and all age groups. Perforation rate was high (41.4\%), and was more prevalent in male (54.9\%) and in the 0-9 age group (65.7\%).

Conclusions: Appendicitis, both acute and chronic, is more distributed in the second decade, and is slightly more prevalent in females. Acute cases are more common than chronic. Perforation rate is significant and peaks in the first decade and in males. [AMJ.2017;4(1):36-41]

Keywords: Acute appendicitis, chronic appendicitis, distribution

\section{Introduction}

Appendicitis is a medical emergency and a common cause of emergency surgery worldwide. ${ }^{1,2}$ Frequency of appendicitis is varied by population, age, sex, socioeconomic status, and race. Untreated appendicitis may be complicated with gangrene formation and perforation, leading to a high morbidity and mortality in almost all age groups. ${ }^{3}$ To prevent morbidity and mortality, which is about $2 \%$ associated with perforation, timely surgery is needed. ${ }^{4}$ However, gold standard for diagnosis of appendicitis is the histopathology examination. ${ }^{5}$

Clinically and histologically, appendicitis is divided into acute and chronic appendicitis. ${ }^{6,7}$ Acute appendicitis is more prevalent in male than female..$^{2-5}$ It is commonly seen in the second decade of age, with lifetime risk of $7 \%{ }^{1-3}$ Chronic appendicitis, in the other hand, is more frequent in female and in $>25$ years of age. ${ }^{8}$ Perforation is more prevalent in male and in the age of 0 to 9 years and $>50$ years. ${ }^{3}$ This study was conducted to explain the distribution pattern of appendicitis according to age, sex, and the histopathologic type.

\section{Methods}

This cross-sectional study was conducted at the Department of Anatomical Pathology, Dr. Hasan Sadikin General Hospital, Bandung, Indonesia, August-October 2013. The secondary data were collected after permission letters had been issued by the Health Research Ethics Committee (Komite Etik Penelitian Kesehatan/ KEPK) and the research site. Medical records from January $1^{\text {st }}$ to December $31^{\text {st }}, 2012$, were collected using total population technique. An inclusion criterion was histopathologic diagnosis of appendicitis. Patient's age, sex,

Correspondence: Tara Zhafira, Faculty of Medicine, Universitas Padjadjaran, Jalan Raya Bandung-Sumedang Km.21, Jatinangor, Sumedang, Indonesia, Phone: +6281311349612 Email: tarazhafira@yahoo.com 
and histopathologic type of appendicitis were noted. Medical record with incomplete data was excluded. The obtained data were then evaluated using a spreadsheet software program.

The patient's age was divided into 7 groups, with a 10 years range for each group while the patient's sex was divided into male and female. The histopathologic types were divided into acute and chronic, based on conclusion of the histopathologic examination noted in the records. Frequency of each group was then calculated. Any perforation and specific cases were also noted.

\section{Results}

There were 516 medical records which fulfilled the inclusion criterion. Among 516 cases, 13 were excluded: 12 due to incompleteness of data (there were no age information in 5 cases, no sex information in 7 cases) and 1 case was considered inappropriate for the evaluation (the age recorded was 5 month and too extreme for the evaluation). A final 503 cases were included for the review.

Furthermore, the age of the population was ranged from 1 to 81 years, with a mean (SD) of 28.1 (15.3) years. The peak incidence of appendicitis occurred in the 10 to 19 years group, counting for $28.4 \%$ of all cases. There was $52.3 \%$ proportion of female, with male: female ratio 1:1. This study also showed 96 $(19.1 \%)$ of the pediatric population (aged 0 to 15 years) was with male predominance (61.5\%) and mean (SD) of 10.7 (3.6) years (Table 1).

In this study, male were more distributed in the $0-9$ and in $\geq 50$ years of age. Females were predominated in the 10-49 years group (Figure 1). Mean age (SD) for male was 28.6 (17.3) and for female was 27.8 (13.2).

There were 495 cases $(98.4 \%)$ of acute appendicitis in this study. Incidence of acute appendicitis was higher than the chronic appendicitis in both sexes and all age groups (Table 2). From 8 chronic cases, 3 were specific for tuberculous appendicitis, consisting of 2 male and 2 patients from the 20 to 29 years group.

According to age, acute appendicitis was more prevalent in the 10 to 19 years group, while chronic appendicitis was higher in the 20 to 29 years group. Both acute and chronic appendicitis were predominated by female. There was $100 \%$ rate of acute appendicitis in the pediatric population aged 0 to 15 years.

Among the acute appendicitis cases, 205 perforated cases were noted (Table 2). This complication was peaked in the 10 to 19 years age group and in male. There were also 59 perforated cases observed in the pediatric population aged 0 to 15 years in this study.

Perforation rate was calculated by dividing

Table 1 Distribution of Appendicitis according to Age, Sex and Histopathologic Type

\begin{tabular}{lcc}
\hline \multicolumn{1}{c}{ Variable } & N & \% \\
\hline Age (Years) & & 7 \\
$0-9$ & 35 & 28.4 \\
$10-19$ & 143 & 26.2 \\
$20-29$ & 132 & 14.9 \\
$30-39$ & 75 & 11.5 \\
$40-49$ & 58 & 7.6 \\
$50-59$ & 38 & 4.4 \\
$\geq 60$ & 22 & \\
Sex & & 47.7 \\
Male & 240 & 52.3 \\
Female & 263 & \\
Histopathologic Type of Appendicitis & & 98.4 \\
Acute & 495 & 1.6 \\
Chronic & 8 & \\
\hline
\end{tabular}




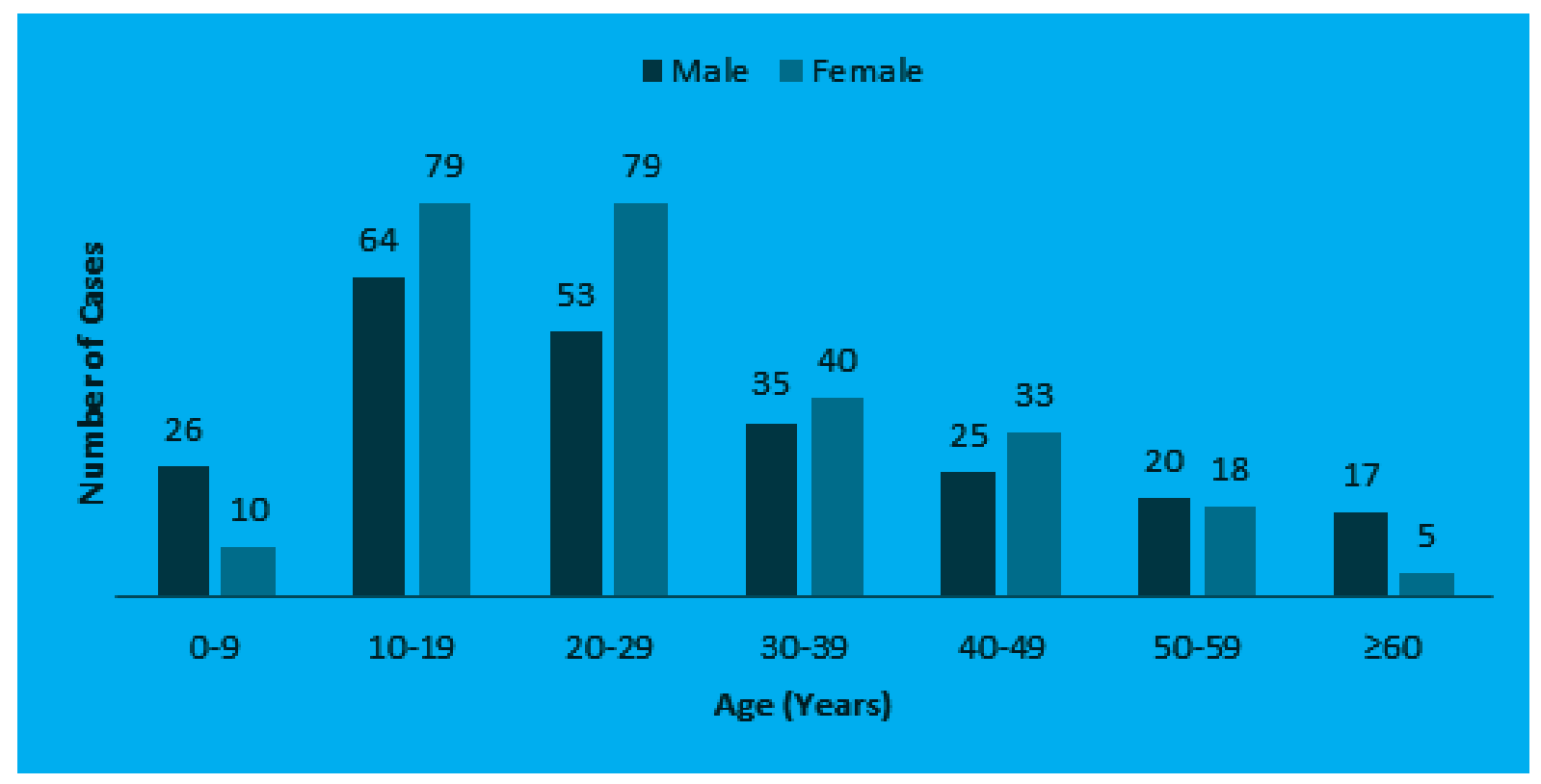

Figure 1 Sex Distribution of Appendicitis according to Age

numbers of perforated cases by numbers of acute cases. With 205 perforated cases and 495 acute cases, the total perforation rate was $41.4 \%$. Perforation rate peaked at $65.7 \%$ and was observed in the 0 to 9 years age group (Figure 2). There was also perforation rate of $60.8 \%$ observed in the pediatric population. According to sex, perforation rate of male $(54.9 \%)$ was higher than female $(29.1 \%)$.
Gradual decrease of perforation rate was observed from the first to the third decade $(65.7 \%$ to $28.7 \%)$.

\section{Discussions}

The results showed a peak incidence of appendicitis in the 10 to 19 years group. Additionally, similar results were obtained

Table 2 Mann Whitney Analysis Result

\begin{tabular}{lccccc}
\hline \multirow{2}{*}{ Variable } & \multicolumn{2}{c}{ Acute } & Perforation & \multicolumn{2}{c}{ Chronic } \\
\cline { 2 - 6 } & $\mathbf{n}$ & $\mathbf{\%}$ & $\mathbf{n}$ & $\mathbf{n}$ & $\mathbf{\%}$ \\
\hline Age (years) & 35 & 7.1 & 23 & 0 & 0 \\
$0-9$ & 142 & 28.7 & 63 & 1 & 12.5 \\
$10-19$ & 129 & 26.1 & 37 & 3 & 37.5 \\
$20-29$ & 73 & 14.7 & 33 & 2 & 25 \\
$30-39$ & 57 & 11.5 & 23 & 1 & 12.5 \\
$40-49$ & 37 & 7.5 & 17 & 1 & 12.5 \\
$50-59$ & 22 & 4.4 & 9 & 0 & 0 \\
$\geq 60$ & & & & & \\
Sex & 237 & 47.9 & 130 & 3 & 37.5 \\
Male & 258 & 52.1 & 75 & 5 & 62.5 \\
Female & & & & & \\
\hline
\end{tabular}




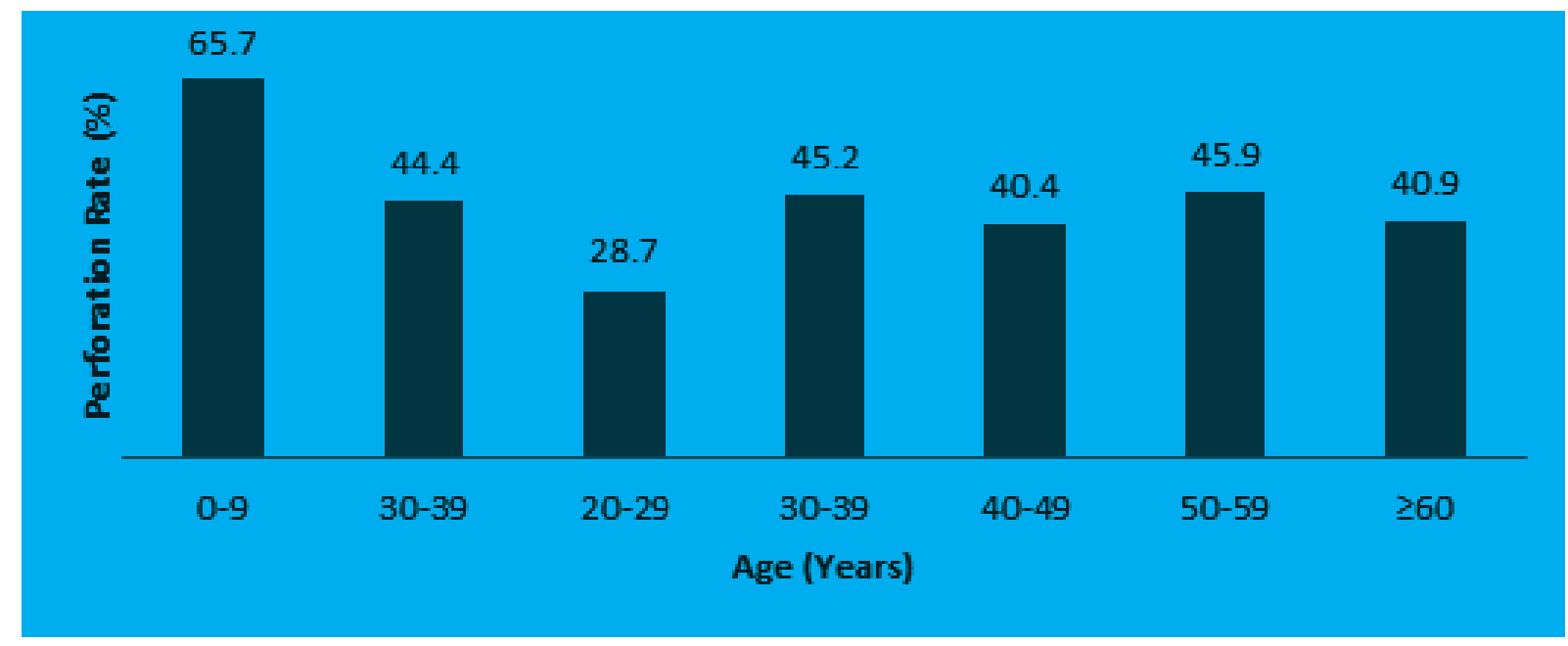

Figure 2 Perforation Rate according to Age

by Chamisa et al. ${ }^{2}$, Sulu et al. ${ }^{3}$, Kalliakmanis et al. $^{9}$ and Chandrasegaram et al. ${ }^{10}$ Those series stated that appendicitis is higher in the second decade. However, different results are concluded by Makaju et al. ${ }^{4}$ and Zulfikar et al. ${ }^{11}$ There is a higher prevalence of appendicitis in the 21 to 30 years age group in their studies.

Besides, female was more prevalent in this study. Similar results were observed by Kalliakmanis et al. ${ }^{9}$, Namsawang et al. ${ }^{12}$, Shrestha et al. ${ }^{13}$ and Sgourakis et al. ${ }^{18}$ However, there were also some series that revealed opposite findings. ${ }^{2-5,10,11}$

Histopathologic examination is a gold standard for diagnosing appendicitis. ${ }^{5}$ It serves two purposes, which are to confirm the diagnosis and to disclose any additional pathology that may be unseen in surgeries but can affect the management. ${ }^{11}$ Besides, clinically and histopathologically, appendicitis is divided into acute and chronic appendicitis. ${ }^{6,7}$

Acute appendicitis is marked by neutrophil infiltration in the appendiceal wall. Based on the pathologic stages, acute appendicitis can be further divided into early acute, acute suppurative, acute gangrenous, and perforative., ${ }^{4,6,15}$ Early acute appendicitis is macroscopically defined by dull serosal surface of appendix. Microscopically, it shows perivascular and mild to moderate transmural neutrophil infiltration. Acute suppurative appendicitis is marked macroscopically by serosal fibrinopurulent exudates and microscopically by dense transmural neutrophilic infiltration with mucosal ulceration. Acute gangrenous is marked grossly by greenish-black necrosis. It shows extensive mucosal ulceration and hemorrhage necrosis of all layers under the microscope. ${ }^{4,15}$ This grouping of acute appendicitis, however, was not applied in our study site at the time of this study was conducted. The histopathologic type of appendicitis was defined as acute and chronic only.

In this study, acute appendicitis was more prevalent than chronic appendicitis. Some studies concluded similar results, with the incidence of chronic appendicitis ranged from $0.58 \%$ to $6.55 \%{ }^{4,11,14,16}$ This study found that the incidence of acute appendicitis peaked in the 10 to 19 years group and was gradually decreasing afterwards, consistent with the result concluded by Chandrasegaram et al. ${ }^{10}$ Female population was predominant both in acute and chronic appendicitis.

The existence of chronic appendicitis is controversial, but Safaei et al. ${ }^{8}$ concluded that it should be considered as an independent diagnostic entity. On histopathologic examination, it shows inflammatory infiltrates consisting of lymphocytes, histiocytes, and eosinophils with fibrosis of the appendiceal wall. ${ }^{8}$ In this study, chronic appendicitis was higher in the 20 to 29 years group. A study conducted by Shrestha et al. ${ }^{13}$ showed similar result, stating chronic appendicitis is more common in the 21 to 40 years group, while Safaei et al. ${ }^{8}$ stated that its incidence is higher in $>25$ years of age and in female.

There were $3(0.59 \%)$ cases of tuberculous appendicitis in this study. Besides, tuberculous appendicitis was also observed by Makaju 
et al. $^{4}$ and Zulfikar et al. ${ }^{11}$ with a rate of $0.58 \%$ and $3 \%$, respectively. Tuberculous appendicitis is rare in the Western world, and is thought to be caused by the poor hygiene and low socioeconomic level in an area. ${ }^{11,17}$ Macroscopically there are inflammation and thickening of the appendiceal wall. It is often adhered to the surrounding bowel with associated lymphadenitis. Histologically, lymphoid hyperplasia and caseating granuloma are observed. Mucosal ulcer can also be seen. Organism is rare, but can be found by staining to reveal the acid fast bacily (AFB). ${ }^{18}$

Furthermore, perforation is one of appendicitis complications and may cause high mortality and morbidity rate than the nonperforated cases.,19 Timely surgery is mandatory to prevent this outcome. ${ }^{15}$ Perforation rate observed in this study was considerably high compared to the other studies which ranged from $0.8 \%$ to $20 \%{ }^{3-5,10,11}$ However, a significant perforation rate (34\%) was also found by Chamisa et al. ${ }^{2}$

Considering the age group, perforated appendicitis peaked in the 0 to 9 years group in our study as shown by Sulu et al. ${ }^{3}$ Besides in the early ages, the literature also stated that perforation is prevalent in the elderly group. Nonspecific symptoms and laboratories, delayed admission, communication problems, and socioeconomic factors were hypothesized to be the cause of higher perforation rate, especially in younger age and elderly., ${ }^{3,19}$ There were two distinctive patterns of perforated appendicitis in this study, consistent with the study conducted by Chandrasegaram et al. ${ }^{10}$ which showed gradual decrease of perforation rate from the first to third decade and an advance of perforation rate with age. The latter can be seen in this study by the consistent high rates of perforation in $>30$ years groups.

Incidence of perforated appendicitis was higher in male in this study, which was consistent with the results concluded by Sulu et al. ${ }^{3}$ and Nabipour et al. ${ }^{5}$ The higher incidence of appendicular faecoliths and calculi found in male was proposed to be an explanation of higher perforation rate in this group. ${ }^{19}$ Studies conducted by Chamisa et al. ${ }^{2}$, Chandrasegaram et al. $^{10}$, Shrestha et al. ${ }^{13}$ and Barreto et al. ${ }^{19}$ however, did not obtain the same outcome. The series observed perforated appendicitis is more commonly seen in female. ${ }^{2,10,13,19}$

Appendicitis is also a common cause of acute abdomen and emergency abdominal surgeries in children. ${ }^{16}$ Among $19.1 \%$ of pediatric population in this study with mean age (SD) of 10.7 (3.6), males were predominant. A study of children appendicitis by Monajemzadeh et al. ${ }^{16}$ revealed mean age (SD) of 6.9 (3.5) and male predominance. However, Uba et al. $^{20}$ observed female predominance in the pediatric population.

The literature stated that the histopathologic type more commonly observed in children was acute appendicitis, with the rate of chronic appendicitis only $0.8 \%{ }^{16}$ This study showed a hundred per cent acute appendicitis in children. Acute appendicitis in early ages is theorized to be caused by lymphoid hyperplasia. ${ }^{3}$ It is characterized by the increasing of lymphoid tissues' size due to immunological reaction against infected agents like virus. Lymphoid hyperplasia alone, however, can cause symptoms. ${ }^{20}$ Interestingly, perforation rate in pediatric population of this study was higher than the results concluded in studies conducted by Monajemzadeh et al. ${ }^{16}$ and Uba et al. ${ }^{20}$ which are $13.8 \%$ and $25 \%$, respectively. Delay of diagnosis caused by children's inability to communicate precisely the explanation for their symptoms is considered to be the cause of high perforated cases in children. ${ }^{16}$

This study was limited by the less specific histopathologic classification of acute appendicitis. Therefore, the histopathologic results could not be presented based on the pathologic stages, which are early acute, acute suppurative, and acute gangrenous.

In conclusion, appendicitis is more distributed in the 10 to 19 years group and in females. Acute appendicitis is more commonly encountered than chronic appendicitis in both sexes and all age groups. There is a considerably high perforation rate, which peaks in the 0 to 9 years group and in males. A further study of appendicitis distribution in another site is recommended.

\section{References}

1. Kumar V, Abbas AK, Fausto $\mathrm{N}$, Aster JC. Pathologic Basis of Disease. 8th ed. Philadelphia: Saunders Elsevier; 2005. p. 826-7.

2. Chamisa I. A clinicopathological review of 324 appendices removed for acute appendicitis in Durban, South Africa. Ann R Coll Surg Engl. 2009;91(8):688-92.

3. Sulu B, Gunerhan Y, Palanci Y, Isler B, Caglayan K. Epidemiological and demographic features of appendicitis and influences of several environmental factors. Ulus Travma Acil Cerrahi Derg. 2010;16(1):38-42. 
4. Makaju R, Mohammad A, Shakya A. Acute appendicitis: analysis of 518 histopathologically diagnosed cases at the Kathmandu University Hospital, Nepal. Kathmandu Univ Med J. 2010;8(30):22730 .

5. Nabipour F. Histopathologic feature of acute appendicitis in Kerman-Iran From 1997-2003. Am J Environ Sci. 2005;1(2):130-2.

6. Rosai J. Rosai and Ackerman's Surgical Pathology. 10th ed. Edinburgh: Mosby Elsevier; 2011. p. 714-6

7. Berger DH, Jaffe BM. Schwartz's Principles of Surgery. $9^{\text {th }}$ ed. New York: McGraw-Hill; 2010. p. 1073-89.

8. Safaei M, Moeinei L, Rasti M. Recurrent abdominal pain and chronic appendicitis. J Res Med Sci. 2004;9(1):11-4.

9. Kalliakmanis V, Pikoulis E, Karavokyros I, Felekouras E, Morfaki P, Haralambopoulou G, et al. Acute appendicitis: the reliability of diagnosis by clinical assessment alone. Scand J Surg. 2005;94(3):201-6.

10. Chandrasegaram MD, Rothwell LA, Ethan I, Miller RJ. Pathologies of the appendix: a 10-year review of 4670 appendectomy specimens. ANZ J Surg. 2012;82(11):8447.

11. Zulfikar I, Khanzada TW, Sushel C, Samad A. Review of the pathologic diagnoses of appendectomy specimens. Annals of King Edward Medical University. 2009;15(4):168-70.

12. Namsawang P. Pathological findings of vermiform appendix in Surin Hospital. Medical Journal of Srisaket Surin Buriram
Hospitals. 2009;24(1):199-208.

13. Shrestha R, Ranabhat SR, Tiwari M. Histopathologic analysis of appendectomy specimens. Journal of Pathology of Nepal. 2012;2(3):215-9.

14. Sgourakis G, Sotiropoulos GC, Molmenti EP, Eibl C, Bonticous S, Moege J, et al. Are acute exacerbations of chronic inflammatory appendicitis triggered by coprostasis and/or coprolith? World J Gastroenterol. 2008;14(20):3179-82.

15. Sujata J, Zeeba J, Siafia R. Appendicitishistology specific incidence trends in a semi urban population in New Delhi: a study of 262 cases. Bangladesh J Med Sci. 2012;11(3):201-205

16. Monajemzadeh M, Hagghi-Ashtiani M, Montaser-Kouhsari L, Ahmadi H. Pathologic evaluation of appendectomy specimens in children. Iran J Pediatr. 2011;21(4):485-90.

17. Igho OE. Unusual histological findings of the appendix following appendectomy, a ten year histopathologic study. Continental J Biomedical Sciences. 2012;6(1):41-5.

18. Lamps LW. Infectious causes of appendicitis. Infect Dis Clin North Am. 2010;24(4):995-1018.

19. Barreto SG, Travers E, Thomas T, Mackillop $\mathrm{C}$, Tiong L, Lorimer $\mathrm{M}$, et al. Acute perforated appendicitis: an analysis of risk factors to guide surgical decision making. Indian J Med Sci. 2010;64(2):58-65.

20. Uba A, Lohfa E, Ayuba M. Childhood acute appendicitis: is routine appendicectomy advised? J Indian Assoc Pediatr Surg. 2006;11(1):27-30. 